WISSENSCHAFTSZENTRUM BERLIN FÜR SOZIALFORSCHUNG

SOCIAL SCIENCE RESEARCH CENTER BERLIN

\author{
Kai A. Konrad * \\ Dan Kovenock **
}

Equilibrium and Efficiency in the Tug-of-War

* WZB and Free University of Berlin

** Purdue University

SP || $2005-14$

August 2005

ISSN Nr. $0722-6748$

Research Area

Markets and Political Economy

Research Unit

Market Processes and Governance
Forschungsschwerpunkt Markt und politische Ökonomie

Abteilung

Marktprozesse und Steuerung 
Zitierweise/Citation:

Kai A. Konrad, Dan Kovenock, Equilibrium and Efficiency in the Tug-of-War, Discussion Paper SP II 2005 - 14, Wissenschaftszentrum Berlin, 2005.

Wissenschaftszentrum Berlin für Sozialforschung gGmbH, Reichpietschufer 50, 10785 Berlin, Germany, Tel. (030) 25491 - 0 Internet: www.wz-berlin.de 


\title{
ABSTRACT
}

\section{Equilibrium and Efficiency in the Tug-of-War}

\author{
by Kai A. Konrad and Dan Kovenock *
}

We characterize the unique Markov perfect equilibrium of a tug-of-war without exogenous noise, in which players have the opportunity to engage in a sequence of battles in an attempt to win the war. Each battle is an all-pay auction in which the player expending the greater resources wins. In equilibrium, contest effort concentrates on at most two adjacent states of the game, the "tipping states", which are determined by the contestants' relative strengths, their distances to final victory, and the discount factor. In these states battle outcomes are stochastic due to endogenous randomization. Both relative strength and closeness to victory increase the probability of winning the battle at hand. Patience reduces the role of distance in determining outcomes.

Applications range from politics, economics and sports, to biology, where the equilibrium behavior finds empirical support: many species have developed mechanisms such as hierarchies or other organizational structures by which the allocation of prizes are governed by possibly repeated conflict. Our results contribute to an explanation why. Compared to a single stage conflict, such structures can reduce the overall resources that are dissipated among the group of players.

Keywords: Winner-take-all, all-pay auction, tipping, multi-stage contest, dynamic game, preemption, conflict, dominance

JEL Classification: D72, D74

* Comments by Daniel Krähmer and Johannes Münster are gratefully acknowledged. Part of this work was completed while the second author was Visiting Professor at the Social Science Research Center Berlin (WZB). The usual caveat applies. 


\section{Gleichgewicht und Effizienz im "Tug of War"}

Wir beschreiben das eindeutige Markoff-perfekte Gleichgewicht in einem mehrstufigen Konflikt ohne exogene Unsicherheit ("noise"), bei dem die Spieler versuchen, in einer Serie von aufeinander folgenden kleineren Gefechten einen Konflikt zu gewinnen. Jedes Gefecht ist eine "all-pay auction", bei der derjenige Spieler gewinnt, der die meisten Ressourcen eingesetzt hat. Im Gleichgewicht konzentriert sich der Mitteleinsatz auf höchstens zwei benachbarte Zustände, die wir als spielentscheidende Zustände ("tipping states") bezeichnen. Die Lage dieser Zustände hängt von der relativen Stärke der Spieler, der Zahl der verbleibenden Spielstufen bis zum Gesamtsieg und dem Diskontierungsfaktor ab. An diesen kritischen Zuständen ist der Konfliktausgang zufällig aufgrund der stochastischen Verteilung der im Gleichgewicht gewählten Mengen von Konfliktressourcen. Sowohl die relative Stärke als auch die Nähe zur finalen Konfliktstufe erhöhen die Wahrscheinlichkeit, das einzelne Gefecht zu gewinnen. Geringe Kosten des Wartens verringern den Einfluss der Entfernung zum Gesamtsieg auf den Ausgang der einzelnen Gefechte. Die Anwendungsgebiete sind zahlreich und reichen von der Politik über die Wirtschaft und den Sport bis zur Biologie. Dort findet das Gleichgewichtsergebnis empirisch Unterstützung: Viele Arten haben eigene Mechanismen entwickelt, z.B. Hierarchien oder andere Organisationsstrukturen, bei denen die Allokation der Siegerprämie in sich möglicherweise wiederholenden Konflikten erfolgt. Unsere Ergebnisse liefern hierzu eine Erklärung. Im Vergleich mit einem einstufigen Konflikt können solche Strukturen den Ressourceneinsatz der Spieler reduzieren. 


\section{Introduction}

Final success or failure in a conflict is often the result of the outcomes of a series of potential battles. An illustrative example is the decision making process in many organizations. Resources, jobs and other goods that involve rents to individuals inside the organization are frequently allocated in a process that has multiple decision stages. For instance, hiring decisions often involve a contest between candidates in which a hiring committee makes a decision and forwards this decision to another committee. This committee approves to the initial decision and forwards the case further until a final decision stage is reached, or may return the case to the previous committee. Candidates could expend effort trying to influence the decision process in each stage, but if at all, typically serious efforts are expended by the candidates only in early stages of the decision process. Such multi-layered decision processes obviously cause delay in decision making and this can be seen as a cost. We will argue here that, compared to a single stage decision process in which the rival players spend effort in a single stage all-pay auction, the multi-stage decision process can be advantageous as it may improve allocative efficiency and reduce effort that is expended by rival contestants in the conflict.

In more general terms we describe the multi-stage contest as a tug-ofwar. As a modeling device, the tug-of-war has a large number of applications in diverse areas of science, including political science, economics, astronomy, history and biology. ${ }^{1}$ It consists of a (possibly infinite) sequence of battles between two contestants who accumulate stage victories, and in which the

\footnotetext{
${ }^{1}$ To give a few examples: In politics, Whitford (2005) describes the struggle between the president and legislature about the control of agencies as a tug-of-war. Yoo (2001) refers to the relations between the US and North-Korea and Organski and Lust-Okar (1997) to the struggle about the status of Jerusalem as cases of tug-of-war. According to Runciman (1987), at the time of the Crusades, when various local rulers frequently attacked one another, they sometimes succeeded in conquering a city or a fortification, only to lose this, or another, part of their territory to the same, or another, rival ruler later on. The conflict between two rival rulers can be seen as a sequence of battles. They start at some status quo in which each rules over a number of territories with fortified areas. They fight each other in battles, and each battle is concerned with one fortress or territory. In the sequence of successes and failures, the fortresses or territories are destroyed or reallocated, and the conflict continues until one of the rulers has lost all his fortresses or territories and is thus finally defeated. If battle success alternates more or less evenly, then such a contest can go on for a very long time, possibly even forever. The end comes only when one of the rulers has been more successful than his rival sufficiently often.
} 
contestant who first accumulates a sufficiently larger number of such victories than his rival is awarded the prize for final victory. ${ }^{2}$

To our knowledge, Harris and Vickers (1987) were the first to look formally at the tug-of-war. They analyse an $R \& D$ race as a tug-of-war in which each single battle is determined as the outcome of a contest with noise. Such exogenous noise makes the problem less tractable and has so far ruled out a fully analytic description of the equilibrium. Budd, Harris and Vickers (1993) apply a somewhat more complicated stochastic differential game approach to a dynamic duopoly, seen as a tug-of-war involving a continuum of advertizing or R\&D battles that determine the firms'relative market positions. Using a complementary pair of asymptotic expansions for extreme parameter values and numerical simulations elsewhere, they isolate a number of effects that govern the process. Several of these appear in our analysis which, unlike their framework, derives an analytical solution for the unique Markov perfect equilibrium. Morever, our analysis explicitly solves for equilibrium for both symmetric and asymmetric environments.

The term 'tug-of-war' has also been used in biology. In the context of within-group conflict among animals, subjects could struggle repeatedly. ${ }^{3}$ For instance, the formation of hierarchies and their dynamic evolution occurs in repeated battle contests. As Hemelrijk (2000) describes for several examples, individuals may try to acquire a high rank, but the differentiation and asymmetry that is created by this can also reduce future conflict. Winning or losing a particular contest in a series of conflictual situations is known to change future conflict behavior (Bergman et al. (2003), Beacham (2003) and Hsu and Wolf (1999)). This may partially be the result of information about own fighting skills and the fighting experience gained, but it may also arise

\footnotetext{
${ }^{2}$ According to Wikipedia the term tug-of-war refers to a rope pulling contest in which two contestants (or groups) pull a rope in different directions until one of the sides pulled the rope (and the opponent group) across a certain limit. In more abstract terms, the contest consists of a series of battles, where a battle victory of one player makes both move one unit towards the winner's preferred terminal state, and where one contestant wins the war if the difference between the winner's number of such battle victories exceeds the other contestant's number of battle victories by some absolute number.

${ }^{3}$ The term also refers to contests between different species. Ehrenberg and McGrath (2004) refer to the interaction of microtubule motors, Larsson, Beignon and Bhardwaj (2004) and Zhou et al. (2004) refer to the interaction between viruses and the dendritic cells or other parts of the immune system as tugs of war. Tibbetts and Reeve (2000) consider the role of the amount of reproductive sharing within a group for the likelihood of within-group conflict among the social wasp Polistes dominulus.
} 
from the change in strategic position with respect to future conflict about rank, territory, access to food, or opportunities to reproduce.

Evidence from biology and political science shows that violent conflict often does not take place, or, at least, the intensity of a conflict varies significantly as a function of the conflicting parties' actual strengths, previous experience, and the strategic symmetry or asymmetry of the particular situation in terms of territorial or other advantages. Parker and Rubenstein (1981) and Hammerstein (1981) emphasize the role of asymmetry in determining whether a conflictual situation turns into a resource wasteful or violent conflict. Different advantages and disadvantages may determine the overall asymmetry of a conflictual situation, and counterbalance or add to each other. Schaub (1995) describes the conflict over food that occurs between long-tailed macaque females. Differences in strength and in the distances between the animals and the location of the food govern their behavior. Superior strength or dominance of one contestant can be compensated by a greater distance she has to the location of the food. Relative strength, together with the actual payoffs from winning determine contestants' stakes at any given stage of a tug-of-war and determine the degree of asymmetry between the rival players.

We examine how the players' respective fighting abilities, rewards from final victory, and the distances in terms of the required battle win differential to achieve victory interact to determine Markov perfect equilibrium behavior in the tug-of-war. For notational convenience we concentrate on the asymmetry in the valuations of the final prize and assume equal fighting ability, but as will be shown this is equivalent to the more general case with asymmetric valuations of the prize and asymmetric fighting abilities. We show that the contest effort that is dissipated in total and over all battle periods crucially depends on the starting point of the tug-of-war, and, for many starting points, is negligible, even if the asymmetry in the starting conditions is very limited. Hence, the multi-battle structure in a tug-of-war reduces the amount of resources that is dissipated in the contest, compared to a single all-pay auction, which has been studied by Hillman and Riley (1989) and Baye, Kovenock and deVries $(1993,1996)$ for the case of complete information and by Amann and Leininger (1995, 1996), Krishna and Morgan (1997), Kura (1999), Moldovanu and Sela (2001) and Gavious, Moldovanu and Sela (2002) in the context of incomplete information. ${ }^{4}$

\footnotetext{
${ }^{4}$ For further applications of the all-pay auction see Arbatskaya (2003), Baik, Kim and
} 
Our results may contribute to explaining why mechanisms such as hierarchies or other organizational structures have evolved by which the allocation of prizes is governed by a multi-stage conflict. ${ }^{5}$ Such structures may delay the allocation of a given prize, compared to a single stage conflict, but can considerably reduce the overall resources that are dissipated among the group of players. Compared to a standard all-pay auction, a tug-of-war that is not rigged in favor of one of the players also improves allocative efficiency; the probability with which the prize is awarded to the player who values it more highly is higher in the tug-of-war than in the standard all-pay auction.

In the next section we outline the structure of the tug-of-war and characterize the unique Markov perfect equilibrium. In section 3 we discuss the efficiency properties of the tug-of-war and compare it with the all-pay auction. Section 4 concludes.

\section{The analytics of the tug-of-war}

A tug-of-war is a multi-stage game with a potentially infinite horizon which is characterized by the following elements. The set of players is $\{A, B\}$. The set of states of the war is given by a finite ordered grid of $m+1$ points $M \equiv\{0,1, \ldots m\}$ in $R^{1}$. The tug-of-war begins at time $\mathrm{t}=1$ with players in the intitial state $j(1)=m_{A}, 0<m_{A}<m$, which may either be chosen by nature, or may be a feature of the institutional design. In each period $t=1,2,3 \ldots$ a battle takes place between the players in which $A$ (resp. $B)$ expends effort $a_{t}$ (resp. $b_{t}$ ). A victory by player $A(B)$ in state $i$ at time $t$ moves the war to state $i-1(i+1)$ at time $t+1$. The state in period $t+1$ is therefore $j(t+1)=m_{A}+n_{B t}-n_{A t}$, where $n_{A t}$ and $n_{B t}$ denote respectively,

$\mathrm{Na}$ (2001), Baye, Kovenock and De Vries (2005), Che and Gale (1998, 2003), Ellingsen (1991), Kaplan, Luski and Wettstein (2003), Konrad (2004), Moldovanu and Sela (2004), and Sahuguet and Persico (2005).

${ }^{5}$ There are, of course, other explanations for hierarchies more generally, which, however, focus on different aspects of a hierarchy (see, e.g., the survey in Radner 1992). Radner (1993) for instance, considers a problem of efficient information aggregation, asking what is the efficient decision tree. Closer to the issue of allocation of goods in a conflict, Wärneryd (1998) and Müller and Wärneryd (2001) consider distributional conflict between rival groups followed by distributional conflict within the winning group as a type of hierarchical conflict. Both these approaches focus on the "tree-ör "pyramidproperty of hierarchies that reduces the number of players when moving to the top, whereas our approach does not use this property. We consider only two contestants throughout and focus on the sequential, repeated nature of decision process. 
the number of battle victories that $A$ and $B$ have accumulated by the end of period $t$. This continues as long as the war stays in some interior state $j \in M^{i n t} \equiv\{1,2, \ldots,(m-1)\}$. The war ends when one of the players achieves final victory by driving the state to his favored terminal state, $j=0$ and $j=m$, for player $A$ and $B$ respectively. A prize (for final victory) of size $Z_{A}$ is awarded to $A$ if the terminal state $j=0$ is reached and, alternatively, a prize of $Z_{B}$ is awarded to $B$ if the terminal state $j=m$ is reached. Without loss of generality we assume that $Z_{A} \geq Z_{B}$. Figure 1 depicts the set of states.

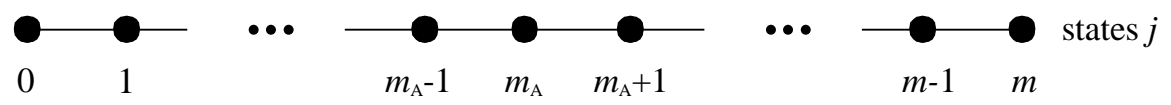

Figure 1:

Player $A$ 's (B's) period $t$ payoff $\pi_{A}\left(a_{t}, j(t)\right)\left(\pi_{B}\left(b_{t}, j(t)\right)\right)$ is assumed to equal $Z_{A}\left(Z_{B}\right)$ if player $A(B)$ is awarded the prize in that period, and $-a_{t}\left(-b_{t}\right)$ if $t$ is a period in which effort is expended. ${ }^{6}$ We assume that each player maximizes the expected discounted sum of his per-period payoffs. Throughout we assume that $0<\delta<1$ denotes the common, time invariant, discount factor. ${ }^{7}$

The assumption that the cost of effort is simply measured by the effort itself is for notational simplicity only. Since a player's preference over income streams is invariant with respect to a positive affine transformation of utility, if player $A(B)$ has a constant unit cost of effort $c_{A}\left(c_{B}\right)$ we may normalize utility by dividing by $c_{A}\left(c_{B}\right)$ to obtain a new utility function representing the same preferences in which the unit cost of effort is 1 but player $A(B)$ has a prize value $Z_{A} / c_{A}\left(Z_{B} / c_{B}\right)$. Therefore, our model with asymmetric prizes can be interpreted as one with both asymmetric prizes and fighting abilities.

Each single battle in the tug-of-war is a simultaneous move all-pay auction with complete information. A player's action in each period in which the state is interior is his effort, $a_{t} \in[0, K]$ and $b_{t} \in[0, K]$, for $A$ and $B$,

\footnotetext{
${ }^{6}$ Since the per-period payoffs do not depend directly on time, we have dropped a time index.

${ }^{7}$ It is straightforward to extend our results to cases in which players have different, time invariant discount factors $\delta_{A}$ and $\delta_{B}$.
} 
respectively, where $K \geq Z_{A}{ }^{8}$ The player who spends the higher effort in a period wins the battle. We choose a deterministic tie-breaking rule for the case in which both players choose the same effort, by which the "advantaged" player wins. Given $m, Z_{A}, Z_{B}$ and $\delta$, we say that player $A$ is advantaged in state $j$ if $\delta^{j} Z_{A}>\delta^{m-j} Z_{B}$, and $B$ is advantaged if $\delta^{j} Z_{A} \leq \delta^{m-j} Z_{B}$. We define $j_{0}=\min \left\{j \in M^{i n t} \mid \delta^{j} Z_{A} \leq \delta^{m-j} Z_{B}\right\}$ where this is non-empty, and $j_{0}=m$ otherwise: player $B$ is advantaged for $j \in M^{\text {int }}$ such that $j \geq j_{0}$ and $A$ is advantaged otherwise.

If $m=2$ and $m_{A}=1$, the tug-of-war reduces to the well-known case of the standard all-pay auction with complete information at time $t=1$, as in Hillman and Riley (1989), Ellingsen (1991) or Baye, Kovenock and deVries (1996). In this case, one single battle takes place at state $j=m_{A}=1$. The process moves from this state in period 1 to $j=0$ or to $j=2$ at the beginning of period 2, and the prize is handed over to $A$ or $B$, respectively. Accordingly, the contest at period $t=1$ in state $j=1$ is over a prize that has a present value of $\delta Z_{A}$ and $\delta Z_{B}$ for $A$ and $B$, respectively, and the payoffs in the unique equilibrium of this game (which are in nondegenerate mixed strategies) are $\delta\left(Z_{A}-Z_{B}\right)$ for $A$ and zero for $B$. In what follows, we consider the case with $m>2$.

For each period $t$, if a terminal state has not yet been reached by the beginning of the period, players simultaneously choose efforts with common knowledge of the initial state $m_{A}$ and the full history of effort choices, denoted as $\left(\mathbf{a}_{t-1}, \mathbf{b}_{t-1}\right) \equiv\left(\left(a_{1}, \ldots, a_{t-1}\right),\left(b_{1}, \ldots, b_{t-1}\right)\right)$. Players also know the current state $j(t)$ of the war and the state in any past period $j(\tau), \tau<t$. We define $\mathbf{j}_{t}=(j(1), j(2), \ldots j(t))$, where $j(1)=m_{A}$. Hence, we will summarize the history at time $t$ along any path which has not yet hit a terminal state by $h^{t}=\left(\mathbf{a}_{t-1}, \mathbf{b}_{t-1}, \mathbf{j}_{t}\right)$. We will call such a path a non-terminal period $t$ history and will denote the set of such histories by $H^{t}$. A history of the game that generates a path that reaches a terminal state at precisely period $t$ is termed a terminal period $t$ history. Denote the set of terminal period $t$ histories by $T^{t}$, and the set of $\left(\mathbf{a}_{t-1}, \mathbf{b}_{t-1}\right)$ generating elements of $T^{t}$ by $T_{e}^{t}$.

If for an infinite sequence of effort choices, $\mathbf{a}=\left(a_{1}, a_{2}, \ldots\right)$ and $\mathbf{b}=$ $\left(b_{1}, b_{2}, \ldots\right)$ no terminal state is reached in finite time, we will call the corresponding history $h^{\infty}=(\mathbf{a}, \mathbf{b}, \mathbf{j})$ a non-terminal history and denote the set

\footnotetext{
${ }^{8}$ This upper limit makes the set of possible effort choices compact, but does not lead to a restriction that could be binding in any equilibrium, as an effort choice larger than $Z_{A}$ in some period is strictly dominated by a choice of effort of zero in this and all future periods.
} 
of such histories as $H^{\infty}$.

Given these constructions, we define a behavior strategy $\sigma_{l}$ for player $l \in\{A, B\}$ as a sequence of mappings $\sigma_{l}\left(h^{t}\right): H^{t} \rightarrow \Sigma_{[0, K]}$, that specifies for every period $t$ and non-terminal history $h^{t}$ an element of the set of probability distributions over the feasible effort levels $[0, K]$. Each behavior strategy profile $\sigma=\left(\sigma_{A}, \sigma_{B}\right)$ generates for each $t$ a probability distribution over histories in the set $\bigcup_{\tau<t} T^{\tau} \cup H^{t}$. It also generates a probability distribution over the set of all feasible paths of the game, $\bigcup_{\tau=1}^{\infty} T^{\tau} \cup H^{\infty}$.

Since we assume that each player's payoff for the tug-of-war is the expected discounted sum of his per-period payoffs, the payoff for player $A$ from a behavioral strategy profile $\sigma$ is denoted $v_{A}(\sigma)=E_{\sigma}\left(\sum_{t=1}^{\tilde{t}} \delta^{t-1} \pi_{A}\left(a_{t}, j(t)\right)\right) \equiv$ $E_{\sigma}\left(\pi_{A}\left(\mathbf{a}_{\tilde{t}-1}, \mathbf{b}_{\tilde{t}-1}, \mathbf{j}_{\tilde{t}}\right)\right)$ where $\tilde{t}$ is the hitting time at which a terminal state is first reached. ${ }^{9}$ If a terminal state is never reached, $\tilde{t}=\infty$. Note that for a given sequence of actions $\left(\mathbf{a}_{\tilde{t}-1} \mathbf{b}_{\tilde{t}-1}\right), \tilde{t}$ arises deterministically, according to the non-random transition rule embodied in the all-pay auction, so that the randomness of $\tilde{t}$ is generated entirely by the non-degenerate nature of the probability distributions chosen by the behavioral strategies. If $h^{t+1}=\left(\mathbf{a}_{t}\right.$ $\left.\mathbf{b}_{t}, \mathbf{j}_{t+1}\right) \in T_{e}^{t+1}$ denotes a sequence of efforts that leads to a terminal state at precisely period $\tilde{t}=t+1$, then, the payoffs for $A$ and $B$ are

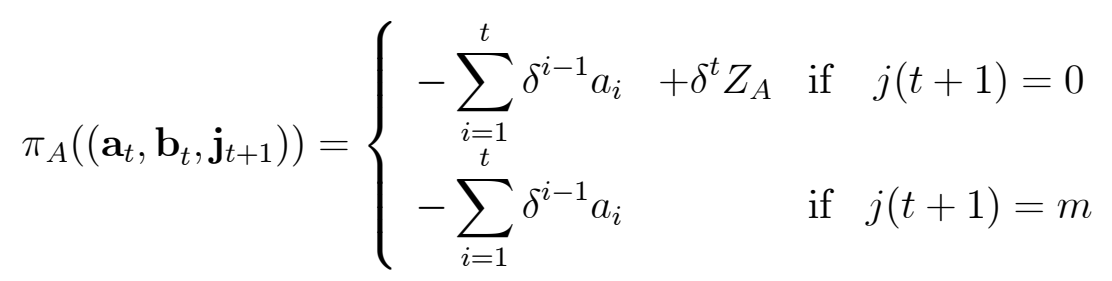

and, respectively,

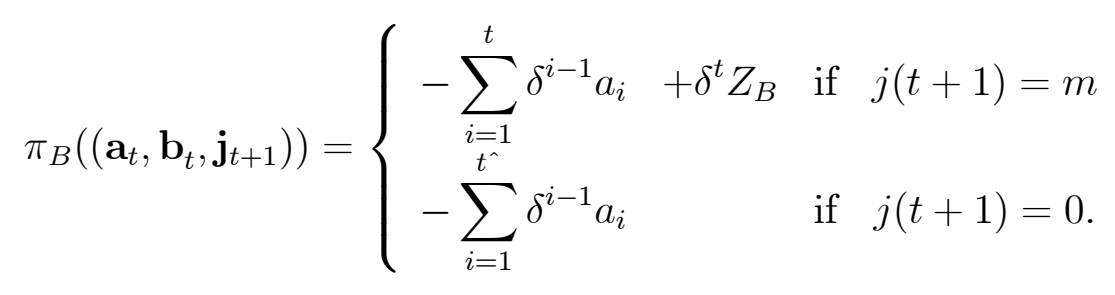

\footnotetext{
${ }^{9}$ We adopt a notational convention throughout this paper that the action set available to each player in a terminal state is the effort level zero, so that for any hitting time $\tilde{t}$, $a_{\tilde{t}}=b_{\tilde{t}}=0$. Hence, in these states $\pi_{A}\left(a_{t}, j\right)$ and $\pi_{B}\left(b_{t}, j\right)$ include only the prize awarded to the victor, and we suppress the terms $a_{\tilde{t}}$ and $b_{\tilde{t}}$ in the notation $\Sigma_{t=1}^{\tilde{t}} \delta^{t-1} \pi_{A}\left(a_{t}, j(t)\right) \equiv$ $\pi_{A}\left(\mathbf{a}_{\tilde{t}-1}, \mathbf{b}_{\tilde{t}-1}, \mathbf{j}_{\tilde{t}}\right)$.
} 
If for an infinite sequence of effort choices, $\mathbf{a}=\left(a_{1}, a_{2}, \ldots\right)$ and $\mathbf{b}=\left(b_{1}, b_{2}, \ldots\right)$ no terminal state is reached in finite time, payoffs are

$$
\pi_{A}((\mathbf{a}, \mathbf{b}, \mathbf{j}))=-\sum_{t=1}^{\infty} \delta^{t-1} a_{t} \quad \text { and } \pi_{B}((\mathbf{a}, \mathbf{b}, \mathbf{j}))=-\sum_{t=1}^{\infty} \delta^{t-1} b_{t} .
$$

For a given behavior strategy profile $\sigma=\left(\sigma_{A}, \sigma_{B}\right)$ each player's payoff in the tug-of-war can be derived from calculating the expected sum of discounted per period payoffs generated by the probability distribution over histories in the set $\bigcup_{\tau=1}^{\infty} T^{\tau} \cup H^{\infty}$. Moreover, for any $t$ and $h^{t} \in H^{t}$, one may define each player's expected discounted value of future per-period payoffs (discounted back to time $t$ ) conditional on the history $h^{t}$ by deriving the conditional distribution induced by $\left.\sigma\right|_{h^{t}}$ over $\bigcup_{\tau=t+1}^{\infty} T^{\tau} \cup H^{\infty}$. We shall refer to this as a player's continuation value conditional on $h^{t}$ and denote it by $v_{i}\left(\sigma \mid h^{t}\right)=$ $E_{\sigma \mid h^{t}}\left(\sum_{s=t}^{\tilde{t}} \delta^{s-t} \pi_{A}\left(a_{s}, j(s)\right)\right)$. Note that this has netted out any expenditures accrued on the history $h^{t}$.

Since the players' objective functions are additively separable in the perperiod (time invariant) payoffs and transitions probabilities depend only upon the current state and actions, continuation payoffs from any sequence of current and future action profiles depend on past histories only through the current state $j$. It therefore seems natural to restrict attention to Markov strategies that depend only on the current state $j$ and examine the set of Markov perfect equilibria. Indeed, this partition of histories is that obtained from the more formal analysis of the determination of the Markov partition in Maskin and Tirole (2001). For any $t$, we may partition past (non-terminal) histories in $H^{t}$ by the period $t$ state $j(t)$, inducing a partition $H^{t}(\cdot)$, and define the collection of partitions, $H(\cdot) \equiv\left\{H^{t}(\cdot)\right\}_{t=1}^{\infty}$. It can be demonstrated that in our game the vector of collections $\left(H^{A}(\cdot), H^{B}(\cdot)\right)=(H(\cdot), H(\cdot))$ is the unique maximally coarse consistent collection (the Markov collection of partitions) in the sense of Maskin and Tirole (2001, p. 201). For any time $t$, the current state $j(t)$ therefore constitutes what they call the payoff-relevant history. Since our game is stationary, we may partition the set of all finite non-terminal histories by the same state variables, $j \in M^{i n t} \equiv\{1,2, \ldots(m-1)\}$, removing any dependence of the partition on the time $t$. We label this partition $\{j(t)=$ $i\}_{i \in M^{i n t}}$. This is the stationary partition defined by Maskin-Tirole, (2001, p. 203).

In the continuation, we restrict attention to (stationary) Markov strategies measurable with respect to the payoff relevant history determined by 
the stationary partition $\{j(t)=i\}_{i \in M^{i n t}}$. A stationary Markov strategy $\sigma_{l}$ for player $l \in\{A, B\}$ is a mapping $\sigma_{l}(j): M^{i n t} \rightarrow \Sigma_{[0, K]}$, that specifies for every interior state $j$ a probability distribution over the set of feasible effort levels $[0, K]$. If in the continuation game starting in period $t$ and state $j, \sigma=\left(\sigma_{A}, \sigma_{B}\right)$ is played, then the continuation value for player $i$ at $t$ is denoted as $v_{i}(\sigma \mid j)$ and can be calculated as the discounted sum of future expected period payoffs in a well-defined manner similar to that described above.

In this context we are interested in deriving the set of Markov perfect equilibria; that is a pair of Markov strategies that constitute mutually best responses for all feasible histories. In Propositions 1-3 below we demonstrate that the tug-of-war has a unique Markov perfect equilibrium for any combination of $m_{A}, m, Z_{A}, Z_{B}$ and $\delta$.

Before stating these propositions, it is useful to derive some simple properties that must hold in any Markov perfect equilibrium of our model. Suppose $\sigma^{*}=\left(\sigma_{A}^{*}, \sigma_{B}^{*}\right)$ is a Markov perfect equilibrium and denote player $i$ 's continuation value in state $j$ under $\sigma^{*}$ by $v_{i}\left(\sigma^{*} \mid j\right)=v_{i}(j)$. Subgame perfection and stationarity imply that competition in any state $j, j \in\{1,2, \ldots m-1\}$, may be viewed as an all-pay auction with prize $z_{A}(j)=\delta v_{A}(j-1)-\delta v_{A}(j+1)$ for player $A$ and $z_{B}(j)=\delta v_{B}(j+1)-\delta v_{B}(j-1)$ for player $B$. In equilibrium, the continuation value to player $l$ of being in state $j$ at time $t$ is equal to the sum of the value of conceeding the prize without a fight (and thereby moving one state away from the player's desired terminal state) and the value of engaging in an all-pay auction with prizes $z_{A}(j)=\delta v_{A}(j-1)-\delta v_{A}(j+1)$ for player $A$ and $z_{B}(j)=\delta v_{B}(j+1)-\delta v_{B}(j-1)$ for player $B$. An immediate consequence of the characterization of the unique equilibrium in the two-player all-pay auction with complete information (see Hillman and Riley (1989) and Baye, Kovenock, and De Vries (1996)) is that local stategies are uniquely determined and the continuation value for the two players in any state $j \in\{1, \ldots m-1\}$ at any time $t$ is

$$
\begin{gathered}
v_{A}(j)=\delta v_{A}(j+1)+\max \left(0, z_{A}(j)-z_{B}(j)\right)=\delta v_{A}(j+1)+ \\
+\max \left(0, \delta\left[\left(v_{A}(j-1)-v_{A}(j+1)\right)-\left(v_{B}(j+1)-v_{B}(j-1)\right)\right]\right)
\end{gathered}
$$

and

$$
\begin{gathered}
v_{B}(j)=\delta v_{B}(j-1)+\max \left(0, z_{B}(j)-z_{A}(j)\right)=\delta v_{B}(j-1)+ \\
+\max \left(0, \delta\left[\left(v_{B}(j+1)-v_{B}(j-1)\right)-\left(v_{A}(j-1)-v_{A}(j+1)\right)\right]\right) .
\end{gathered}
$$


Rearranging (3) and (4) we obtain

$v_{A}(j)=\delta v_{A}(j+1)+\max \left(0, \delta\left[\left(v_{A}(j-1)+v_{B}(j-1)\right)-\left(v_{A}(j+1)+v_{B}(j+1)\right)\right]\right)$

and

$v_{B}(j)=\delta v_{B}(j-1)+\max \left(0, \delta\left[\left(v_{A}(j+1)+v_{B}(j+1)\right)-\left(v_{A}(j-1)+v_{B}(j-1)\right)\right]\right)$

Note that the first summand in (5) and (6) is the discounted value of losing the contest at $j$ and the second summand in each of these expressions is the expected gain arising from the contest at $j$. For at least one player this gain will be zero and for the other player it will be non-negative and strictly positive as long as $J(j-1) \neq J(j+1)$, where $J(l) \equiv v_{A}(l)+v_{B}(l)$ is the joint present value of being in state $l$.

Three immediate implications of the above construction are

(i) $z_{A}(j)-z_{B}(j) \geq 0$ if and only if $J(j-1)-J(j+1) \geq 0$ with strict inequality in one if and only if in the other.

(ii) $z_{A}(j)-z_{B}(j) \geq 0$ if and only if $v_{B}(j)=\delta v_{B}(j-1)$ and $z_{A}(j)-z_{B}(j) \leq 0$ if and only if $v_{A}(j)=\delta v_{A}(j+1)$.

(iii) If $z_{A}(j)-z_{B}(j) \geq 0$ then $\left.v_{A}(j)=\delta\left[v_{A}(j-1)+v_{B}(j-1)\right)-v_{B}(j+1)\right]$, and if $z_{A}(j)-z_{B}(j) \leq 0$ then $\left.v_{B}(j)=\delta\left[v_{A}(j+1)+v_{B}(j+1)\right)-v_{A}(j-1)\right]$.

By assumption 0 and $m$ are terminal states so that $v_{A}(0)=Z_{A} \geq Z_{B}=$ $v_{B}(m)$ and $v_{A}(m)=v_{B}(0)=0$. Moreover, since player $A$ can only receive a positive payoff in the state 0 , player $B$ can only receive a positive payoff in the state $m$, and both players have available the opportunity to always expend zero effort, in any Markov perfect equilibrium the following inequalities hold for all $j$ :

$$
\begin{gathered}
0 \leq v_{A}(j) \leq \delta^{j} Z_{A} \\
0 \leq v_{B}(j) \leq \delta^{m-j} Z_{B}
\end{gathered}
$$

and

$$
v_{A}(j)+v_{B}(j) \leq \max \left(\delta^{j} Z_{A}, \delta^{m-j} Z_{B}\right)
$$


We can now prove the following

Proposition 1 Consider a tug-of-war with $m \geq 3$. Suppose $j_{0} \in\{2, \ldots m-1\}$ exists such that

$$
\delta^{j_{0}-1} Z_{A}>\delta^{m-\left(j_{0}-1\right)} Z_{B} \text { and } \delta^{j_{0}} Z_{A}<\delta^{m-j_{0}} Z_{B} .
$$

Then a unique Markov perfect equilibrium exists which is characterized as follows:

For all interior states $j \notin\left\{j_{0}-1, j_{0}\right\}$, the equilibrium effort choices are $a(j)=b(j)=0$. Only at $j_{0}-1$ and $j_{0}$ does a battle with a positive probability of stricty positive effort choices take place. Payoffs for $A$ in the continuation game at $j$ are $\delta^{j} Z_{A}$ for $j<j_{0}-1, \frac{1}{\left(1-\delta^{2}\right)}\left[\delta^{j_{0}-1} Z_{A}-\delta^{m-\left(j_{0}-1\right)} Z_{B}\right]$ for $j=j_{0}-1$, and 0 for $j \geq j_{0}$; payoffs for $B$ are $\delta^{m-j} Z_{B}$ for $j>j_{0}, \frac{1}{\left(1-\delta^{2}\right)}\left[\delta^{m-j_{0}} Z_{B}-\delta^{j_{0}} Z_{A}\right]$ for $j=j_{0}$ and 0 for $j \leq j_{0}-1$.

Proof. We consider existence here and relegate the proof of uniqueness to the Appendix. We consider the following candidate equilibrium: For all interior states $j \notin\left\{j_{0}-1, j_{0}\right\}$, the effort choices are $a(j)=b(j)=0$. At $j_{0}-1$ and $j_{0}$ players choose efforts according to cumulative distribution functions $F_{j}$ and $G_{j}$ for players $A$ and $B$ in states $j$ as follows:

$$
\begin{aligned}
& F_{j_{0}-1}(a)=\left\{\begin{array}{ccc}
\frac{a}{\frac{\delta}{\left(1-\delta^{2}\right)} \Delta_{B A}^{j_{0}}} & \text { for } & a \in\left[0, \frac{\delta \Delta_{B A}^{j_{0}}}{\left(1-\delta^{2}\right)}\right] \\
1 & \text { for } & a>\frac{\delta \Delta_{B A}^{j_{0}}}{\left(1-\delta^{2}\right)}
\end{array}\right. \\
& G_{j_{0}-1}(b)=\left\{\begin{array}{ccc}
1-\frac{\delta}{\left(1-\delta^{2}\right)} \Delta_{B A}^{j_{0}} & \text { for } & b \in\left[0, \frac{\delta \Delta_{B A}^{j_{0}}}{\left(1-\delta^{2}\right)}\right] \\
1 & \text { for } & b>\frac{\delta \Delta_{B A}^{j_{0}} Z_{A}}{\left(1-\delta^{2}\right)}
\end{array}\right.
\end{aligned}
$$

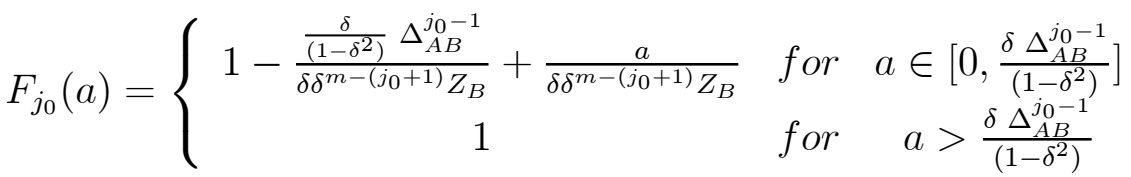

and

$$
G_{j_{0}}(b)=\left\{\begin{array}{cll}
\frac{b}{\frac{\delta}{\left(1-\delta^{2}\right)} \Delta_{A B}^{j_{0}-1}} & \text { for } & b \in\left[0, \frac{\delta \Delta_{A B}^{j_{0}-1}}{\left(1-\delta^{2}\right)}\right] \\
1 & \text { for } & b>\frac{\delta \Delta_{A B}^{j_{0}-1}}{\left(1-\delta^{2}\right)}
\end{array}\right.
$$




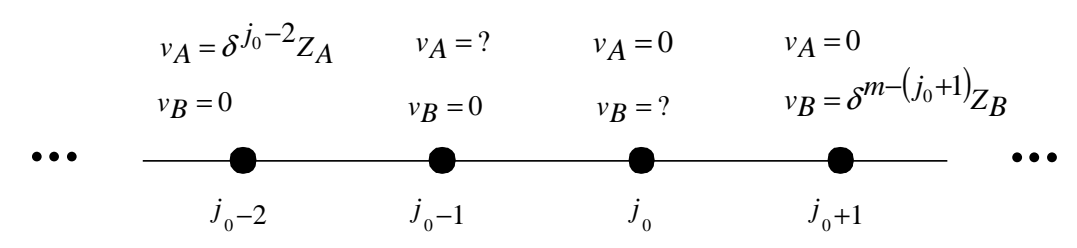

Figure 2:

where

$$
\Delta_{B A}^{j_{0}}=\left[\delta^{m-j_{0}} Z_{B}-\delta^{j_{0}} Z_{A}\right] \text { and } \Delta_{A B}^{j_{0}-1}=\left[\delta^{j_{0}-1} Z_{A}-\delta^{m-\left(j_{0}-1\right)} Z_{B}\right] .
$$

Note first that this equilibrium candidate has the properties described in Proposition 1. Players' continuation values can be stated as functions of the respective state $j$ as follows:

$$
v_{A}(j)=\left\{\begin{array}{ccc}
\delta^{j} Z_{A} & \text { for } & j<j_{0}-1 \\
\frac{1}{\left(1-\delta^{2}\right)}\left[\delta^{j_{0}-1} Z_{A}-\delta^{m-\left(j_{0}-1\right)} Z_{B}\right] & \text { for } & j=j_{0}-1 \\
0 & \text { for } & j \geq j_{0}
\end{array}\right.
$$

and

$$
v_{B}(j)=\left\{\begin{array}{ccc}
\delta^{m-j} Z_{B} & \text { for } & j>j_{0} \\
\frac{1}{\left(1-\delta^{2}\right)}\left[\delta^{m-j_{0}} Z_{B}-\delta^{j_{0}} Z_{A}\right] & \text { for } & j=j_{0} \\
0 & \text { for } & j \leq j_{0}-1 .
\end{array}\right.
$$

These constitute the payoffs stated in the proposition. For $0<j<j_{0}-1$, player $A$ wins the next $j$ battles without any effort. This takes $j$ periods and explains why the value of the final prize must be discounted to $\delta^{j} Z_{A}$. Also, $B$ does not expend effort in these $j$ battles and finally loses after $j$ battles. Hence, $B$ 's payoff is equal to zero. For $m>j>j_{0}$, players $A$ and $B$ simply switch roles.

Turn now to the states $j_{0}-1$ and $j_{0}$ as in Figure 2. We call these states "tipping states", because of their pivotal role in determining the outcome of the contest. Consider $j_{0}-1$. From there, if $A$ wins, the game moves to $j_{0}-2$ with continuation values $v_{A}\left(j_{0}-2\right)=\delta^{j_{0}-2} Z_{A}$ and $v_{B}\left(j_{0}-2\right)=0$. If $B$ wins, the game moves to $j_{0}$ with continuation values $v_{A}\left(j_{0}\right)=0$ and $v_{B}\left(j_{0}\right)$. Assuming that $\delta^{j_{0}-2} Z_{A}>v_{B}\left(j_{0}\right)$ (which can be confirmed later), and applying the results on the standard all-pay auction, the continuation 
values are

$$
v_{A}\left(j_{0}-1\right)=z_{A}\left(j_{0}-1\right)-z_{B}\left(j_{0}-1\right)=\delta\left[\delta^{j_{0}-2} Z_{A}-v_{B}\left(j_{0}\right)\right]
$$

and $v_{B}\left(j_{0}-1\right)=0$, where $z_{A}\left(j_{0}-1\right)$ and $z_{B}\left(j_{0}-1\right)$ denote the prizes that $A$ and $B$ respectively attribute to winning the battle at $j_{0}-1$, given the continuation of the game as described in the candidate equilibrium. Similarly, at $j_{0}$, if $A$ wins, the game moves to $j_{0}-1$ with continuation values $v_{A}\left(j_{0}-1\right)$ as in (18) and $v_{B}\left(j_{0}-1\right)=0$. If $B$ wins, the game moves to $j_{0}+1$ with continuation values $v_{A}\left(j_{0}+1\right)=0$ and $v_{B}\left(j_{0}+1\right)=\delta^{m-\left(j_{0}+1\right)} Z_{B}$. This yields a continuation value for player $B$ of

$$
v_{B}\left(j_{0}\right)=z_{B}\left(j_{0}\right)-z_{A}\left(j_{0}\right)=\delta\left[\delta^{m-\left(j_{0}+1\right)} Z_{B}-v_{A}\left(j_{0}-1\right)\right],
$$

and $v_{A}\left(j_{0}\right)=0$. The solution to this system of equations yields the positive equilibrium values in the middle lines of (16) and (17), and the zero continuation value in the respective state for the other player.

It remains to be shown that the choices described in the candidate equilibrium indeed describe equilibrium behavior. The one-stage deviation principle applies here. ${ }^{10}$ The continuation values (16) and (17) can be used to consider one-stage deviations for $A$ and for $B$.

A deviation $b^{\prime}(j)>0$ at a state $0<j<j_{0}-1$ changes the path from moving to $j-1$ in the next period to $j+1$. However, $v_{B}(j-1)=v_{B}(j+1)=0$. Hence, this deviation reduces $B$ 's payoff by $b^{\prime}(j)$ compared to $b(j)=0$. A deviation $b^{\prime}(j)>0$ at $j>j_{0}$ does not change the state in $t+1$ compared to $b(j)=0$ in the candidate equilibrium, due to the tiebreaking rule employed. The deviation reduces $B$ 's payoff by $b^{\prime}(j)$ compared to $b(j)=0$. An equivalent logic applies for $a(j)$ at states $j \notin\left\{j_{0}-1, j_{0}\right\}$.

Turn now to the state $j_{0}$. In the candidate equilibrium, in state $j_{0}$ contestant $A$ randomizes on the support $\left[0, \frac{\delta}{\left(1-\delta^{2}\right)}\left[\delta^{j_{0}-1} Z_{A}-\delta^{m-\left(j_{0}-1\right)} Z_{B}\right]\right]$. All actions in the equilibrium support for $A$ at $j_{0}$ yield the same expected payoff equal to $G_{j_{0}}(x) \delta v_{A}\left(j_{0}-1\right)+\left(1-G_{j_{0}}(x)\right) 0-x=0$. A possible one-stage deviation for $A$ at $j_{0}$ is an $a^{\prime}\left(j_{0}\right)>\frac{\delta}{\left(1-\delta^{2}\right)}\left[\delta^{j_{0}-1} Z_{A}-\delta^{m-\left(j_{0}-1\right)} Z_{B}\right]$. Compared

\footnotetext{
${ }^{10}$ To confirm this it is sufficient to show that the condition of continuity at infinity is fulfilled for this game. We may then apply Theorem 4.2 in Fudenberg and Tirole (1993). This condition requires that the supremum of the payoff difference that can emerge from strategies that differ after period $t$ converges to zero as $t \rightarrow \infty$. However, a supremum for this is $\delta^{t}\left[Z_{i}+\frac{1}{1-\delta} K\right]$ for $i=A, B$, and this converges to zero as $t \rightarrow \infty$.
} 
to the action $a\left(j_{0}\right)=\frac{\delta}{\left(1-\delta^{2}\right)}\left[\delta^{j_{0}-1} Z_{A}-\delta^{m-\left(j_{0}-1\right)} Z_{B}\right]$ that is inside $A$ 's equilibrium support, this also leads to state $j_{0}-1$, but costs the additional amount $a^{\prime}\left(j_{0}\right)-a\left(j_{0}\right)>0$. The deviation is therefore not profitable for $A$. The same type of argument applies for $b\left(j_{0}\right)$.

A similar argument applies to the state $j_{0}-1$. In the candidate equilibrium, in state $j_{0}-1$ contestant $A$ randomizes on the support $\left[0, \frac{\delta}{\left(1-\delta^{2}\right)}\left(\delta^{m-j_{0}} Z_{B}-\right.\right.$ $\left.\left.\delta^{j_{0}} Z_{A}\right)\right]$. All actions in the equilibrium support for $A$ at $j_{0}-1$ yield the same expected payoff equal to $G_{j_{0}-1}(x) \delta v_{A}\left(j_{0}-2\right)+\left(1-G_{j_{0}-1}(x)\right) 0-x=$ $\frac{1}{1-\delta^{2}}\left[\delta^{j_{0}-1} Z_{A}-\delta^{m-\left(j_{0}-1\right)} Z_{B}\right]=v_{A}\left(j_{0}-1\right) .{ }^{11}$ A possible one-stage deviation for $A$ at $j_{0}-1$ is an $a^{\prime}\left(j_{0}-1\right)>\frac{\delta}{\left(1-\delta^{2}\right)}\left[\delta^{m-j_{0}} Z_{B}-\delta^{j_{0}} Z_{A}\right]$. Compared to the action $a\left(j_{0}-1\right)=\frac{\delta}{\left(1-\delta^{2}\right)}\left[\delta^{m-j_{0}} Z_{B}-\delta^{j_{0}} Z_{A}\right]$ that is the upper bound of $A$ 's equilibrium support, this also leads to state $j_{0}-2$, but costs the additional amount $a^{\prime}\left(j_{0}-1\right)-a\left(j_{0}-1\right)>0$. The deviation is not profitable for $A$. The same type of argument applies for $b\left(j_{0}-1\right)$.

Intuitively, outside of the states $j_{0}-1$ and $j_{0}$, one of the players is indifferent between winning and losing the component contest. For instance, in the state $j_{0}-2$, the best that player $B$ could achieve by winning the next component contest is to enter the state $j_{0}-1$ at which $B$ 's continuation value is still zero and smaller than player $A$ 's continuation value. As $B$ does not gain anything from reaching $j_{0}-1, B$ should not spend any effort trying to reach this state. But if $B$ does not spend effort to win, it is easy for $A$ to win.

The states $j_{0}-1$ and $j_{0}$ are different. Battle victory or defeat at one of these points leads to different continuation games and allocates a considerable rent between $A$ and $B$. This makes competition particularly strong at these states. We call these states "tipping states"because success of an advantaged player at each of these two states "tips"the game so that victory is obtained without further effort. A loss by the advantaged player throws the system back into a competitive state where the player becomes disadvantaged.

Proposition 1 also shows that the allocation of a prize in a tug-of-war leads to a seemingly peaceful outcome whenever the conflict starts in a state other than a tipping state. This will be important for drawing conclusions in section 3 about the efficiency properties of a tug-of-war as an allocation

\footnotetext{
${ }^{11}$ More formally, all actions in the support of $A$ 's equilibrium local strategy that are not mass points of $B$ 's local strategy yield the same expected payoff. Since $B$ has a mass point at zero, this does not hold at $a=0$, but for every $a$ in a neigborhood above zero.
} 
mechanism.

Proposition 1 does not consider all possible parameter cases. Before turning to the remaining cases, note that the case $j_{0}=1$ cannot emerge, as this requires $\delta Z_{A}<\delta^{m-1} Z_{B}$, and this contradicts $Z_{A} \geq Z_{B}$ for $m>2$. However, player $A$ 's dominance could be sufficiently large that no interior $j_{0}$ exists that has the properties defined in Proposition 1. This leads to

Proposition 2 Suppose that $\delta^{m-1} Z_{A}>\delta Z_{B}$. Then a unique Markov perfect equilibrium exists with $v_{B}(j)=0$ and $v_{A}(j)=\delta^{j} Z_{A}$ for all $j \in\{1, \ldots, m-2\}$, and $v_{A}(m-1)=\delta^{m-1} Z_{A}-\delta Z_{B}$ and $v_{B}(m-1)=0$ at $j=m-1$.

Proof. We show that the following effort choices constitute an equilibrium and yield the payoffs described in the proposition. Uniqueness follows the argument in the Appendix.

Effort is $a(j)=b(j)=0$ for all $j \in M^{\text {int }} \backslash\{m-1\}$ and for $j=m-1$ efforts are chosen according to the following cumulative distribution functions:

$$
\begin{gathered}
F_{m-1}(a)=\left\{\begin{array}{ccc}
\frac{a}{\delta Z_{B}} & \text { for } & a \in\left[0, \delta Z_{B}\right] \\
1 & \text { for } & a>\delta Z_{B}
\end{array}\right. \\
G_{m-1}(b)=\left\{\begin{array}{ccc}
\left(1-\frac{\delta Z_{B}-b}{\delta^{m-1} Z_{A}}\right) & \text { for } & b \in\left[0, \delta Z_{B}\right] \\
1 & \text { for } & b>\delta Z_{B} .
\end{array}\right.
\end{gathered}
$$

Note that this behavior yields the payoffs that are characterized in Proposition 2 . For states $j=1,2, \ldots,(m-2), A$ wins after $j$ further battles, and none of the players expends effort. This confirms $v_{A}(j)=\delta^{j} Z_{A}$ and $v_{B}(j)=0$ for all $j=1, \ldots m-2$. For $j=m$, the payoffs are $v_{A}(m)=0$ and $v_{B}(m)=Z_{B}$. Finally, for $j=m-1$, given the mixed strategies described by (20) and (21), the payoffs are $v_{A}(m-1)=\delta^{m-1} Z_{A}>\delta Z_{B}$ and $v_{B}(m-1)=0$.

Now we confirm that the effort choices in the candidate equilibrium are indeed mutually optimal replies. For interior states $j<m-1$, a deviation $b^{\prime}(j)>0$ makes $B$ win the battle, instead of $A$. It leads to $j+1$, instead of $j-1$, but $v_{B}(j+1)=v_{B}(j-1)=0$. Hence, this deviation reduces $B$ 's payoff by $b^{\prime}(j)$ compared to $b(j)=0$. For $A$, for $j<m-1$, contestant $A$ reaches $j=0$ along the shortest possible series of battle victories and does not spend any effort. Any positive effort can therefore only decrease $A$ 's payoff. For $j=m-1$, the battle either leads to $j=m$ where $B$ finally wins the prize, or to $j=m-2$. The values the players attribute to reaching these states are $v_{A}(m)=0, v_{B}(m)=Z_{B}$, and $v_{A}(m-2)=\delta^{m-2} Z_{A}$ and 
$v_{B}(m-2)=0$. Using the results in Hillman and Riley (1989) and Baye, Kovenock and deVries (1996) on a complete information all-pay auction with prizes $\delta\left[\delta^{m-2} Z_{A}-0\right]=\delta^{m-1} Z_{A}$ for $A$ and $\delta\left[Z_{B}-0\right]=\delta Z_{B}$ for $B$, it is confirmed that (20) and (21) describe the unique equilibrium cumulative distribution functions of effort for this all-pay auction.

Proposition 2 shows that a very strong player has a positive continuation value regardless of the interior state in which the tug-of-war starts and wins with probability 1 without expending effort for every interior state except $j=m-1$.

So far we have ruled out the case of equality of continuation values at interior states, and we turn to this case now which exhausts the set of possible cases.

Proposition 3 The tug-of-war with $\delta^{j_{0}} Z_{A}=\delta^{\left(m-j_{0}\right)} Z_{B} \equiv Z$ for some $j_{0} \in$ $\{2, \ldots(m-1)\}$ has a unique subgame perfect equilibrium in which players spend $a(j)=b(j)=0$ in all interior states $j \neq j_{0}$. They choose efforts $a(j)$ and $b(j)$ at $j=j_{0}$ from the same uniform distribution on the range $[0, Z]$. Payoffs are $v_{A}(j)=\delta^{j} Z_{A}$ and $v_{B}(j)=0$ for $j<j_{0}, v_{A}(j)=0$ and $v_{B}(j)=\delta^{m-j} Z_{B}$ for $j>j_{0}$ and $v_{A}(j)=v_{B}(j)=0$ for $j=j_{0}$.

Proof. We again construct an equilibrium to demonstrate existence. Uniqueness follows from arguments similar to those appearing in the Appendix.

In the candidate equilibrium each contestant expends zero effort at any state $j \neq j_{0}$ and expends effort at $j=j_{0}$ according to a draw from the distribution

$$
F(x)=\left\{\begin{array}{ccc}
\frac{x}{Z} & \text { for } & x \in[0, Z] \\
1 & \text { for } & x>Z .
\end{array}\right.
$$

At $j=j_{0}$ the expected effort of each player equals $Z / 2$, and each wins this battle with a probability of $1 / 2$ and, in this case, eventually wins the overall contest $j_{0}-1$ or $\left(m-j_{0}\right)-1$ periods later, respectively, without spending any further effort. This determines the continuation values in the candidate equilibrium. These continuation values are

$$
\begin{array}{ccc}
v_{A}=v_{B}=0 & \text { if } j=j_{0} \\
v_{A}=\delta^{j} Z_{A} \text { and } v_{B}=0 & \text { if } j<j_{0} \\
v_{A}=0 \text { and } v_{B}=\delta^{m-j} Z_{B} & \text { if } & j>j_{0} .
\end{array}
$$

It remains to show that the candidate equilibrium describes mutually optimal replies. Consider one-stage deviations for $A$ and $B$ for some state 
$j<j_{0}$. A choice $a^{\prime}(j)>0$ will not change the equilibrium outcome in the battle in this period and hence will simply reduce $A^{\prime}$ 's payoff by $a^{\prime}(j)$. A choice $b^{\prime}(j)>0$ will make $B$ win. If $j<j_{0}-1$, following the candidate equilibrium $A$ will simply win a series of battles until final victory occurs. Hence, $b^{\prime}(j)>0$ reduces $B$ 's payoff by this same amount $b^{\prime}(0)$ of effort. If $j=j_{0}-1, B$ 's battle victory will lead to $j=j_{0}$, and candidate equilibrium play from here on will yield a payoff equal to zero to $B$. Accordingly, the deviation $b^{\prime}(j)>0$ yields a reduction of $B$ 's payoff by this same amount. Consider one-stage deviations for $A$ and $B$ in some state $j>j_{0}$. The same line of argument applies, with $A$ and $B$ switching roles. Finally, consider one-stage deviations for $A$ and $B$ at $j=j_{0}$. Any such deviation for $A$ must be a choice $a^{\prime}(j)>Z$. Compared to $a(j)=Z$, this choice makes $A$ win with the same probability 1 , but yields a reduction in $A$ 's payoff by $a^{\prime}(j)-Z$, compared to $a(j)=Z$. The same argument applies for deviations by $B$ at this state.

The intuition for Proposition 3 is as follows. The two contestants enter into a very strong fight whenever they reach the state $j=j_{0}$. In this state they are perfectly symmetric and they anticipate that the winner of the battle in this state moves straight to final victory. In the battle that takes place in this case, they dissipate the maximum feasible rent from winning this battle. This maximum rent is what they get if they can move from there through a series of uncontested battles to final victory. Once one of the contestants, say $A$, has acquired some advantage in the sense that the contest has moved to $j<j_{0}$, the only way for $B$ to reach victory passes through the state with $j=j_{0}$. As all rent is dissipated in the contest that takes place there, $B$ is simply not willing to spend any effort to move the contest to that state. Hence, the considerable effort that is spent at the point at which the tug-ofwar becomes symmetric in terms of the prizes that are at stake for the two contestants prevents the contestant who is lagging behind in terms of battle victories from spending positive effort.

Discounting played two important roles in our analysis. First, discounting leads to payoff functions that are continuous at infinity, allowing the application of the one-stage deviation principle, which greatly facilitates our proofs. Moreover, discounting is essential in giving a meaningful role to the distance to the state with final victory. The following holds:

Proposition 4 For a given value of $\frac{Z_{A}}{Z_{B}}>1$, the tipping state $j_{0}$ is an increasing step function of $\delta$. Moreover, as $\delta \rightarrow 1$, A wins the tug-of-war without 
effort starting from any state $j<m-1$.

Proof. The tipping state $j_{0}$ is by definition the smallest state $j$ for which player $B$ is advantaged: $j_{0}=\min \left\{j \in M^{i n t} \mid \delta^{j} Z_{A} \leq \delta^{m-j} Z_{B}\right\}$ when this set is non-empty, and $j_{0}=m$ otherwise. For $\delta>0$, the inequality $\delta^{j} Z_{A} \leq \delta^{m-j} Z_{B}$ is equivalent to $\delta^{2 j-m} Z_{A} \leq Z_{B}$. Since $m \geq 3$, for $\delta$ sufficently close to zero the inequality is clearly satisfied for $j=m-1$, so that $j_{0}$ is interior. Moreover, since $\delta^{2 j-m} \geq 1$ for $j \leq \frac{m}{2}$, it must be the case that $j_{0}>\frac{m}{2}$. As $\delta \rightarrow 1$, the inequality is violated at all interior states, even at $j=m-1$. In this case, by definiton $j_{0}=m$, and from Proposition 2 player $A$ wins the war from any state $j<m-1$. For any $0<\delta<1, \delta^{2 j-m} Z_{A} \leq Z_{B}$ is equivalent to $2 j-m \geq \frac{\log \frac{Z_{B}}{Z_{A}}}{\log \delta}$, so that $j_{0}$ is the smallest index $j$ satisfying the inequality. Since the left hand side of this inequality is positive, and both the numerator and denominator of the right hand side are negative, as $\delta$ increases, the right hand side monotonically increases, eventually diverging to $\infty$ as $\delta \rightarrow 1$. Hence, as $\delta$ increases, the smallest index $j$ satisfying the inequality must increase in steps until it hits $m$.

As the discount factor increases, relative prize value or player strength plays a greater role in the determination of the outcome than distance. For any given value of $\frac{Z_{B}}{Z_{A}}<1$, as $\delta$ increases the tipping state $j_{0}$ moves in discrete jumps towards $m$. Player $A$ may suffer a greater distance disadvantage and still win the prize with certainty.

\section{Expenditure, allocative efficiency and the cost of delay}

The tug-of-war with $m>2$ resolves the allocation problem along a sequence of states, where a violent battle may, but need not take place at each state. Only in the tipping states is positive effort expended with positive probability. Once the process leaves the tipping states, the war moves to a terminal state, without further effort being expended. A tug-of-war that starts in a tipping state will therefore be called "violent". A tug-of-war that starts outside a tipping state will be called "peaceful".

Compared to the standard all-pay auction, the tug-of-war could be interpreted as an institution that saves cost of effort in the problem of allocating a prize between rivals who are prepared to expend resources in fighting for the 
prize. Suppose for instance that $Z_{A}$ and $Z_{B}$ are independent random draws from a continuous distribution with support $[0, \kappa]$, where $\kappa<K$. Suppose that these values are known to the contestants but are not observable by the designer of the institution at the time that it must be implemented. Consider the following tug-of-war as an anonymous mechanism in the case in which $m$ is an even number, so that $\frac{m}{2}$ is integer valued. Start the tug-of-war in the symmetric state $\frac{m}{2}$ and assume, as we have throughout, that player $A$ attempts to move the state to $j=0$ and player $B$ attempts to move the state to $j=m$. Then the following result derives the probability of peaceful resolution:

Proposition 5 Let $\Gamma(g)$ be the continuous cumulative distribution function of $g \equiv Z_{A} / Z_{B}$, with support $[0, \infty]$. The allocation is peaceful in the Markov perfect equilibrium with a probability $\Gamma\left(\delta^{2}\right)+\left(1-\Gamma\left(\frac{1}{\delta^{2}}\right)\right)$, and $\lim _{\delta \rightarrow 1}\left(\Gamma\left(\delta^{2}\right)+\right.$ $\left.\left(1-\Gamma\left(\frac{1}{\delta^{2}}\right)\right)\right)=1$.

Proof. For a proof we show that $\Gamma\left(\delta^{2}\right)+\left(1-\Gamma\left(\frac{1}{\delta^{2}}\right)\right)$ is the probability that the symmetric state $m / 2$ is not a tipping state. Suppose that $g>\frac{1}{\delta^{2}}$. Then $Z_{A}>Z_{B}$ and $\delta^{\frac{m}{2}+1} Z_{A}>\delta^{m-\left(\frac{m}{2}+1\right)} Z_{B}$. Hence, $j_{0}-1>\frac{m}{2}$. By Propositions 1-3 this implies that the tug-of-war that starts in $m / 2$ consecutively moves to $j=0$ with no effort being expended. Let $g<\delta^{2}$. Then $Z_{A}<Z_{B}$. Applying the results in Propositions 1-3 with $A$ and $B$ and $j=0$ and $j=m$ switching roles shows that the tug-of-war that starts in $m / 2$ moves to $j=m$ with no effort being expended. Suppose now that $g \in\left(1, \frac{1}{\delta^{2}}\right)$. In this case $j_{0}-1=m / 2$. Accordingly, a battle with positive expected efforts takes place at $m / 2$ if $\delta^{\frac{m}{2}+1} Z_{A} \neq \delta^{m-\left(\frac{m}{2}+1\right)} Z_{B}$. No expected effort is expended in this case only if $\delta^{\frac{m}{2}+1} Z_{A}=\delta^{m-\left(\frac{m}{2}+1\right)} Z_{B}$. However, the set of values of $g$ for which $\delta^{\frac{m}{2}+1} Z_{A}=$ $\delta^{m-\left(\frac{m}{2}+1\right)} Z_{B}$ holds has a measure of zero. A similar argument applies for $g \in\left(\delta^{2}, 1\right)$, again with $A$ and $B$ and $j=0$ and $j=m$ switching roles.

Proposition 5 characterizes conditions on the asymmetry in the valuations of the prize that are sufficient to make the tug-of-war evolve peacefully if it starts in the symmetric state $j=m / 2$. A sufficient condition for this to happen is that $j=\frac{m}{2}$ is not a tipping state. If tipping states are $j_{0}$ and $j_{0}-1$ with $j_{0}-1>m / 2$ then the equilibrium process moves from $j=\frac{m}{2}$ further away from the tipping states towards the terminal state $j=0$. If $Z_{A}<Z_{B}$, and, hence, tipping states are $j_{0}$ and $j_{0}+1$, with $j_{0}+1<m / 2$, then the equilibrium process moves further away from these states and towards the terminal state $j=m$. 
Note that the number of states is irrelevant for whether the tug-of-war that starts in state $j=\frac{m}{2}$ is resolved peacefully or not, provided that $m>2$. Whether the tug-of-war is resolved peacefully or not depends only on the ratio of the two prizes and the discount factor. For a given continuous distribution of $g$, as the discount factor becomes large, the tug-of-war is resolved almost surely peacefully.

Of course, offsetting the potential gains from the tug-of-war in promoting the peaceful resolution of resource contests are the potential costs of delay arising from the multi-stage nature of the conflict. The all-pay auction is resolved in a single stage $(m=2)$ and hence reduces this delay to the minimum attainable in a non-trivial contest. On the other hand, from Proposition 5 it is apparent that adding more states beyond $m=4$ does not increase the chance of peaceful resolution and only adds potential delay when a peaceful outcome arises. Moreover, if $\frac{m}{2}$ is a tipping state, for a given draw of $Z_{A}$ and $Z_{B}$ the sum of expected payoffs at this state is simply

$$
\frac{\delta^{m / 2}}{1-\delta^{2}} \max \left\{\left(Z_{A}-Z_{B}\right),\left(Z_{B}-Z_{A}\right)\right\}
$$

which is a strictly decreasing function in $m$. We state this as

Proposition 6 The sum of expected payoffs in the tug-of-war with $m \geq 4$ which have a symmetric state $\frac{m}{2}$ is maximized at $m=4$.

Using Proposition 5 we may compare the cases $m=2$ and $m=4$. When $m=4$ we know that there is a probability $\Gamma\left(\delta^{2}\right)+\left(1-\Gamma\left(\delta^{-2}\right)\right)$ that the allocation is peaceful and a probability of $\Gamma\left(\delta^{-2}\right)-\Gamma\left(\delta^{2}\right)$ that the allocation is violent. We know that in the case $m=2$ the allocation is always violent and the sum of the players' payoffs is $\delta\left(Z_{(1)}-Z_{(2)}\right)$, where $Z_{(1)} \equiv \max \left(Z_{A}, Z_{B}\right)$ and $Z_{(2)} \equiv \min \left(Z_{A}, Z_{B}\right)$. Ignoring discounting, the loss due to conflict, $Z_{(2)}$, can be decomposed into the expected loss due to effort expended, $\frac{1}{2} Z_{(2)}[1+$ $\left.\left(Z_{(2)} / Z_{(1)}\right)\right]$, and the expected loss due to misallocation of the prize, $\frac{1}{2} Z_{(2)}[1-$ $\left(Z_{(2)} / Z_{(1)}\right)$ ]. The loss due to delay then comes when the factor $\delta$ is applied.

In the case where $m=4$ the allocation is peaceful when $\left(Z_{(1)} / Z_{(2)}\right)>\delta^{-2}$. In this case, starting from the state $\frac{m}{2}=2$ it takes two periods for the player with the higher value to win and no effort is expended. Hence, the sum of the payoffs of the two players in this case is $\delta^{2} Z_{(1)}$. The only inefficiency in this case is due to delay. For realizations of $\left(Z_{A}, Z_{B}\right)$ satisfying $\left(Z_{(1)} / Z_{(2)}\right)>\delta^{-2}$, the tug-of-war is more efficient than the all-pay auction if and only if $\delta^{2} Z_{(1)}>$ 
$\delta\left(Z_{(1)}-Z_{(2)}\right)$ or, equivalently, $\left(Z_{(1)} / Z_{(2)}\right)<(1-\delta)^{-1}$. Since by assumption we are in the range where the tug-of-war is peaceful, $\left(Z_{(1)} / Z_{(2)}\right)>\delta^{-2}$. Note that $\delta^{-2}>(1-\delta)^{-1}$ if $\delta<\delta^{+} \equiv \frac{\sqrt{5}-1}{2}$. In this case, the all-pay auction is more efficient than the tug-of-war in this range of values of $\left(Z_{(1)} / Z_{(2)}\right)$. For $\delta>\delta^{+}, \delta^{-2}<(1-\delta)^{-1}$ and the tug-of-war is more efficient than the all-pay auction for values of $\left(Z_{(1)} / Z_{(2)}\right)$ in the interval $\left(\delta^{-2},(1-\delta)^{-1}\right)$ and the all-pay auction is more efficient for values of $\left(Z_{(1)} / Z_{(2)}\right)$ in the interval $\left((1-\delta)^{-1}, \infty\right)$.

When $1 \leq\left(Z_{(1)} / Z_{(2)}\right)<\delta^{-2}$, in the tug-of-war the state $\frac{m}{2}$ is a tipping state and the allocation involves active effort expenditure. The expected sum of the payoffs in this case can again be compared to those in the all-pay auction. For $m=4$ the expected sum of the payoffs in the tug-of-war can be calculated from equation $(24)$ and is equal to $\frac{\delta^{2}}{1-\delta^{2}}\left(Z_{(1)}-Z_{(2)}\right)$. Comparing this to the expected sum of payoffs in the all-pay auction we find that for $Z_{(1)} \neq Z_{(2)}, \frac{\delta^{2}}{1-\delta^{2}}\left(Z_{(1)}-Z_{(2)}\right)>\delta\left(Z_{(1)}-Z_{(2)}\right)$ if and only if $\delta^{2}+\delta-1>0$ or $\delta>\delta^{+} \equiv \frac{\sqrt{5}-1}{2}$. Therefore, when the realization of $\left(Z_{A}, Z_{B}\right)$ is such that the initial state $\frac{m}{2}$ is a violent state in the tug-of-war, the tug-of-war is more efficient than the all-pay auction when $\delta>\delta^{+}$and the all-pay auction is more efficient than the tug-of-war when $\delta<\delta^{+}$.

We may summarize these results in the following proposition:

Proposition 7 When $\delta<\delta^{+}=\frac{\sqrt{5}-1}{2}$, for any realization of $\left(Z_{A}, Z_{B}\right)$, the all-pay auction $(m=2)$ is more efficient than a tug-of-war with $m=4$. When $\delta>\delta^{+}$the tug-of-war with $m=4$ is more efficient than the all-pay auction for $\left(Z_{A}, Z_{B}\right)$ such that $\frac{Z_{(1)}}{Z_{(2)}} \in\left(1,(1-\delta)^{-1}\right)$ and the all-pay auction is more efficient for $\frac{Z_{(1)}}{Z_{(2)}} \in\left((1-\delta)^{-1}, \infty\right)$. In particular, for any given continuous joint distribution of $\left(Z_{A}, Z_{B}\right)$, for sufficiently large $\delta$ the tug-or-war is more efficient than the all-pay auction.

\section{Conclusions}

We studied the strategic behavior of players who compete in a series of single battles. A prize is allocated as a function of the sequence of battle successes. A sufficient lead in the number of battle victories is needed to win the final prize. We showed that there is a unique subgame perfect equilibrium in Markov strategies and we characterized this equilibrium. Contest effort concentrates on at most two states. Such states are characterized by three factors: the 
'distance' which the two contestants need to win the overall contest, the relative strength or dominance of contestants, and the discount factor. The critical distance that determines the tipping states in which the contest effort is focussed turns out to be a function of the contestants' relative strengths (or, equivalently, in the relative valuations of the prize from final victory) and the discount factor. The larger one player's dominance in strength, the higher must be this player's distance to final victory, compared to the other player's distance.

Many animal species and economic institutions have developed mechanisms such as hierarchies, or other organizational structures to govern the allocation of prizes, such as preferential food access and the right to reproduce in the biological context, or prized jobs and contracts in the organizational context. Behavior in these mechanisms could be interpreted as a conflict that consists of a series of battles, or repeated opportunities to struggle. Our results help explain why these structures may have evolved. The tug-of-war delays the allocation of a given prize, compared to a single stage conflict, but can considerably increase the efficiency of allocation of the prize and reduce the overall resources that are dissipated among the group of players.

\section{Appendix}

Consider a tug-of-war with $m \geq 3$ and $j_{0} \in\{2, \ldots, m-1\}$ with the property that $\delta^{j_{0}} Z_{A}<\delta^{m-j_{0}} Z_{B}$ and $\delta^{j_{0}-1} Z_{A}>\delta^{m-\left(j_{0}-1\right)} Z_{B}$. Then the Markov perfect equilibrium characterized in the Proposition 1 is unique in the class of Markov perfect equilibria.

We will demonstate the uniqueness of continuation values for every state $j$. For given state-contingent continuation values we have already argued that the problem reduces to a standard all-pay auction for both players at each interior state. Hence, uniqueness results from the uniqueness of the equilibrium in the standard two-player all-pay auction with complete information.

Our proof will start by assuming that $m \geq 3$ and $j_{0} \in\{2, \ldots m-1\}$. (The case of $m=2$ corresponds trivially to the all-pay auction.) We claim the following:

Claim 8 In any Markov perfect equilibrium, for all $k \leq j_{0}-1, v_{B}(k)=0$ and for all $k \leq j_{0}-2, v_{A}(k)=\delta^{k} Z_{A}$. 
Proof. At $k=0$ by construction $v_{A}(0)=Z_{A}$ and $v_{B}(0)=0$, so the claim holds for $k=0$. Since $Z_{A} \geq Z_{B}$ and $m \geq 3$, from (9) evaluated at $k=2$ it follows that $J(0)=Z_{A}>\max \left(\delta^{2} Z_{A}, \delta^{m-2} Z_{B}\right) \geq v_{A}(2)+v_{B}(2)=J(2)$. Hence from $(i) z_{A}(1)>z_{B}(1)$ and from (3) and (4) $v_{A}(1)=\delta\left[Z_{A}-v_{B}(2)\right]$ and $v_{B}(1)=0$. It immediately follows that $J(1)=\delta\left[Z_{A}-v_{B}(2)\right]$. This implies that the claim holds when $j_{0}=2$, which by definition of $j_{0}$ implies that $m=3$.

So assume that $j_{0} \in\{3, \ldots m-1\}$. We will now prove the claim by induction on $k$. Suppose that for some $k, 1 \leq k \leq j_{0}-2, v_{B}(l)=0$ for all $l \leq k$ and $v_{A}(l)=\delta^{l} Z_{A}$ for all $l \leq k-1$. (Note that the supposition holds for $k=1$ ) We claim that $v_{A}(k)=\delta^{k} Z_{A}$ and $v_{B}(k+1)=0$.

To demonstrate this observe that by $(5)$

$v_{A}(k)=\delta v_{A}(k+1)+\max \left(0, \delta\left[\left(v_{A}(k-1)+v_{B}(k-1)\right)-\left(v_{A}(k+1)+v_{B}(k+1)\right)\right]\right)$

Since $v_{B}(k)=0$, by $(6) z_{B}(k)-z_{A}(k)=\delta\left[\left(v_{A}(k+1)+v_{B}(k+1)\right)-\right.$ $\left.\left(v_{A}(k-1)+v_{B}(k-1)\right)\right] \leq 0$, which implies by $(i i i)$ that

$$
\left.v_{A}(k)=\delta\left[v_{A}(k-1)+v_{B}(k-1)\right)-v_{B}(k+1)\right]=\delta\left[\delta^{k-1} Z_{A}-v_{B}(k+1)\right]
$$

Moreover, $v_{B}(k+1)=\delta v_{B}(k)+\delta \max \left(0,\left(v_{A}(k+2)+v_{B}(k+2)\right)-\left(v_{A}(k)+\right.\right.$ $\left.\left.v_{B}(k)\right)\right)=\delta \max \left(0,\left(v_{A}(k+2)+v_{B}(k+2)\right)-\delta\left[\delta^{k-1} Z_{A}-v_{B}(k+1)\right]\right)$.

Suppose by way of contradiction that $v_{B}(k+1)>0$. Then $v_{B}(k+1)=$ $\delta\left[v_{A}(k+2)+v_{B}(k+2)-\delta\left(\delta^{k-1} Z_{A}\right)+\delta v_{B}(k+1)\right]>0$, which implies that $v_{A}(k+2)+v_{B}(k+2)-\delta^{k} Z_{A}=\left(\delta^{-1}-\delta\right) v_{B}(k+1)>0$. However, by (9) $v_{A}(k+2)+v_{B}(k+2) \leq \max \left(\delta^{k+2} Z_{A}, \delta^{m-(k+2)} Z_{B}\right)$. Moreover, by definition of $j_{0}, \delta^{j_{0}-1} Z_{A}>\delta^{m-\left(j_{0}-1\right)} Z_{B}$, which, since $k+2 \leq j_{0}$, implies that $\delta^{k+2-1} Z_{A}>$ $\delta^{m-(k+2)+1} Z_{B}$, or $\delta^{k} Z_{A}>\delta^{m-(k+2)} Z_{B}$. This in turn implies that $v_{A}(k+2)+$ $v_{B}(k+2) \leq \max \left(\delta^{k+2} Z_{A}, \delta^{m-(k+2)} Z_{B}\right)<\delta^{k} Z_{A}$,contradicting the claim that $v_{A}(k+2)+v_{B}(k+2)-\delta^{k} Z_{A}=\left(\delta^{-1}-\delta\right) v_{B}(k+1)>0$ Hence, $v_{B}(k+1)=0$, which immediately implies that $v_{A}(k)=\delta v_{A}(k-1)=\delta^{k} Z_{A}$.

This induction argument therefore shows for all $k \leq j_{0}-1, v_{B}(k)=0$ and for all $k \leq j_{0}-2, v_{A}(k)=\delta^{k} Z_{A}$. An immediate consequence is that

$$
v_{A}\left(j_{0}-1\right)=\delta\left[\left(v_{A}\left(j_{0}-2\right)+v_{B}\left(j_{0}-2\right)\right)-v_{B}\left(j_{0}\right)\right]=\delta\left[\delta^{j_{0}-2} Z_{A}-v_{B}\left(j_{0}\right)\right]
$$


This equation will be used in the continuation to derive values at $j_{0}-1$ and $j_{0}$.

To address equilibrium behavior in states greater than or equal to $j_{0}$,we start in state $m$. Note that since $m$ is a terminal state $v_{B}(m)=Z_{B}$ and $v_{A}(m)=0$.If $j_{0}=m-1$, then by definition $\delta^{m-1} Z_{A}<\delta Z_{B}$ and $\delta^{m-2} Z_{A}>$ $\delta^{2} Z_{B}$. Moreover, by Claim $1 v_{B}(m-2)=0$ and by $(25) v_{A}(m-2)=\delta^{m-2} Z_{A}-$ $\delta v_{B}(m-1)$. Hence,

$$
\begin{aligned}
v_{A}(m-1) & =\delta v_{A}(m)+\delta \max \left(0, v_{A}(m-2)+v_{B}(m-2)-v_{A}(m)-v_{B}(m)\right) \\
& =0+\delta \max \left(0, J(m-2)-Z_{B}\right) \\
& \leq \delta \max \left(0, \max \left(\delta^{m-2} Z_{A}, \delta^{2} Z_{B}\right)-Z_{B}\right) \\
& \leq 0
\end{aligned}
$$

where the final inequality follows from the fact that $\delta^{m-2} Z_{A}=\delta^{j_{0}-1} Z_{A}<$ $\delta^{m-j_{0}-1} Z_{B}=\delta^{m-(m-1)-1} Z_{B}=Z_{B}$. Hence, $v_{A}(m-1)=0$. It follows that

$$
v_{B}(m-1)=\delta v_{B}(m-2)+\delta \max \left(0, Z_{B}-v_{A}(m-2)-v_{B}(m-2)\right)=\delta\left(Z_{B}-v_{A}(m-2)\right)
$$

Hence,

$$
\begin{aligned}
& v_{A}(m-2)=\delta^{m-2} Z_{A}-\delta v_{B}(m-1) \quad \text { and } \\
& v_{B}(m-1)=\delta\left(Z_{B}-v_{A}(m-2)\right)
\end{aligned}
$$

Solving simultaneously implies

$$
v_{A}(m-2)=\frac{\delta^{m-2} Z_{A}-\delta^{2} Z_{B}}{1-\delta^{2}}>0
$$

and

$$
v_{B}(m-1)=\frac{\delta Z_{B}-\delta^{m-1} Z_{A}}{1-\delta^{2}}>0
$$

where both inequalities follow from $j_{0}=m-1$.

This completes the case for $j_{0}=m-1$. So suppose $j_{0} \in\{3, \ldots, m-2\}$ and look at $k>j_{0}$.

Claim 9 In any subgame perfect equilibrium in stationary Markov strategies, for all $k \geq j_{0}, v_{A}(k)=0$ and for all $k \geq j_{0}+1, v_{B}(k)=\delta^{m-k} Z_{B}$. 
Proof. By an argument similar to that above, $v_{B}(m)=Z_{B}$ and $v_{A}(m)=$ 0 imply $J(m)>J(m-2)$, which in turn implies $v_{A}(m-1)=0+\delta \max (0, J(m-$ 2) $\left.-Z_{B}\right)=0$.

Suppose now that for some $k, j_{0}+1 \leq k<m, v_{A}(l)=0$ for all $l \geq k$ and $v_{B}(l)=\delta^{m-l} Z_{B}$ for all $l \geq k+1$. We will now demonstrate that this implies that $v_{B}(k)=\delta^{m-k} Z_{B}$ and $v_{A}(k-1)=0$. Since the supposition holds for $k=m-1$, this will then prove claim 2 by induction.

So assume that for some $k, j_{0}+1 \leq k<m, v_{A}(l)=0$ for all $l \geq k$ and $v_{B}(l)=\delta^{m-l} Z_{B}$ for all $l \geq k+1$. Since $v_{A}(k)=0$, we know that $v_{A}(k-1)+v_{B}(k-1)-v_{A}(k+1)-v_{B}(k+1) \leq 0$, so that

$$
\begin{aligned}
v_{B}(k) & =\delta v_{B}(k-1)+\delta \max \left(0, v_{A}(k+1)+v_{B}(k+1)-v_{A}(k-1)-v_{B}(k-1)\right) \\
& =\delta\left[v_{A}(k+1)+v_{B}(k+1)-v_{A}(k-1)\right] \\
& =\delta\left[\delta^{m-(k+1)} Z_{B}-v_{A}(k-1)\right]
\end{aligned}
$$

Moreover, $v_{A}(k-1)=\delta v_{A}(k)+\delta \max \left(0, v_{A}(k-2)+v_{B}(k-2)-v_{A}(k)-v_{B}(k)\right)$. Since $v_{A}(k)=0$ by assumption and $v_{B}(k)=\delta^{m-k} Z_{B}-\delta v_{A}(k-1)$, we have $v_{A}(k-1)=\delta \max \left(0, v_{A}(k-2)+v_{B}(k-2)-\delta^{m-k} Z_{B}+\delta v_{A}(k-1)\right)$. Suppose by way of contradiction that $v_{A}(k-1)>0$. Then

$$
v_{A}(k-1)=\delta\left[v_{A}(k-2)+v_{B}(k-2)-\delta^{m-k} Z_{B}+\delta v_{A}(k-1)\right]>0 .
$$

or

$$
v_{A}(k-1)\left[\delta^{-1}-\delta\right]=v_{A}(k-2)+v_{B}(k-2)-\delta^{m-k} Z_{B}>0 .
$$

where the last expression is greater than zero because $\delta<1$.

However, from equation $(9), v_{A}(k-2)+v_{B}(k-2) \leq \max \left(\delta^{k-2} Z_{A}, \delta^{m-k+2} Z_{B}\right)$. Since $k \geq j_{0}+1$ implies $k-2 \geq j_{0}-1$, and by definition of $j_{0}, \delta^{j_{0}} Z_{A}<$ $\delta^{m-j_{0}} Z_{B}$, we know $\delta^{j_{0}-1} Z_{A}<\delta^{m-j_{0}-1} Z_{B}$, and hence $\delta^{k-2} Z_{A} \leq \delta^{j_{0}-1} Z_{A}<$ $\delta^{m-j_{0}-1} Z_{B} \leq \delta^{m-k} Z_{B}$. Hence, since both $\delta^{k-2} Z_{A}$ and $\delta^{m-k+2} Z_{B}$ are strictly less than $\delta^{m-k} Z_{B}$, we have a contradiction to $v_{A}(k-2)+v_{B}(k-2)-\delta^{m-k} Z_{B}>$ 0 . Hence, $v_{A}(k-1)=0$. It immediately follows that $v_{B}(k)=\delta^{m-k} Z_{B}$.

We have hence showed by induction that for every $k \geq j_{0}, v_{A}(k)=0$ and for every $k \geq j_{0}+1, v_{B}(k)=\delta^{m-k} Z_{B}$. An immediate consequence is that

$v_{B}\left(j_{0}\right)=\delta v_{B}\left(j_{0}-1\right)+\delta \max \left(0, v_{B}\left(j_{0}+1\right)+v_{A}\left(j_{0}+1\right)-v_{A}\left(j_{0}-1\right)-v_{B}\left(j_{0}-1\right)\right)$ 
Since $v_{A}\left(j_{0}\right)=0$ implies $J\left(j_{0}-1\right)-J\left(j_{0}+1\right) \leq 0$, the maximand in the expression is nonnegative and

$$
\text { (7) } \begin{aligned}
v_{B}\left(j_{0}\right) & =\delta\left[v_{B}\left(j_{0}+1\right)+v_{A}\left(j_{0}+1\right)-v_{A}\left(j_{0}-1\right)\right] \\
& =\delta\left[\delta^{m-\left(j_{0}+1\right)} Z_{B}-v_{A}\left(j_{0}-1\right)\right]
\end{aligned}
$$

Since from $(25) v_{A}\left(j_{0}-1\right)=\delta\left[\delta^{j_{0}-2} Z_{A}-v_{B}\left(j_{0}\right)\right]$, we have a system of two linearly independent equations in two unknowns. These have a unique solution which is

$$
\begin{aligned}
v_{A}\left(j_{0}-1\right) & =\frac{\delta^{j_{0}-1} Z_{A}-\delta^{m-j_{0}+1} Z_{B}}{1-\delta^{2}}>0 \\
v_{B}\left(j_{0}\right) & =\frac{\delta^{m-j_{0}} Z_{B}-\delta^{j_{0}} Z_{A}}{1-\delta^{2}}>0
\end{aligned}
$$

\section{References}

Amann, E. and W. Leininger, 1995, Expected revenue of all-pay and firstprice sealed bid auctions with affiliated signals, Journal of Ecnomics - Zeitschrift für Nationalökonomie 61 (3), 273-279

Amann, E. and W. Leininger, 1996, Asymmetric all-pay auctions with incomplete information: The two-player case, Games and Ecnomic Behavior, 14 (1), 1-18.

Arbatskaya, M., 2003, The exclusion principle for symmetric multi-prize all-pay auctions with endogenous valuations, Economics Letters, 80 (1), 7380 .

Baik, K.H., I.G. Kim and S.Y. Na, 2001, Bidding for a group-specific public-good prize, Journal of Public Economics, 82 (3), 415-429.

Baye, M.R., D. Kovenock, and C. de Vries, 1993, Rigging the lobbying process: an application of the all-pay auction, American Economic Review, 83(1), 289-294.

Baye, M.R., D. Kovenock, and C. de Vries, 1996, The all-pay auction with complete information, Economic Theory, 8(2), 291-305.

Baye, M.R., D. Kovenock, and de Vries, 2005, Comparative analysis of litigation systems: an auction-theoretic approach, Economic Journal (forthcoming). 
Beacham, J.L., 2003, Models of dominance hierarchy formation: Effects of prior experience and intrinsic traits, Behaviour, 140, 1275-1303 Part 10

Bergman, D.A., C. Kozlowski, J.C. McIntyre, R. Huber, A.G. Daws and P.A. Moore, 2003, Temporal dynamics and communication of winner-effects in the crayfish, orconectes rusticus, Behaviour, 140, 805-825 Part 6.

Budd, C., C. Harris and J. Vickers, 1993, A model of the evolution of duopoly: does the asymmetry between firms tend to increase or decrease? Review of Economic Studies, 60(3), 543-573.

Che, Y.K., and I. Gale, 1998, Caps on political lobbying, American Economic Review, 88, 643-651.

Che, Y.K., and I. Gale, 2003, Optimal design of research contests, American Economic Review, 93 (3), 646-671.

Ehrenberg, M. and J.L. McGrath, 2004, Actin mobility: staying on track takes a little more effort, Current Biology, 14(21), R931-R932.

Ellingsen, T., 1991, Strategic buyers and the social cost of monopoly, American Economic Review, 81(3), 648-657.

Fudenberg, D., and J. Tirole, 1993, Game Theory, MIT Press, Cambridge MA.

Gavious, A., B. Moldovanu and A. Sela, 2002, Bid costs and endogenous bid caps, RAND Journal of Economics, 33 (4), 709-722.

Hammerstein, P., 1981, The role of asymmetries in animal contests, Animal Behavior, 29, 193-205.

Harris, C., and J. Vickers, 1987, Racing with uncertainty, Review of Economic Studies, 54(1), 1-21.

Hemelrijk, C.K., 2000, Towards the integration of social dominance and spatial structure, Animal Behaviour, 59, Part 5, 1035-1048.

Hillman, A., and J.G. Riley, 1989, Politically contestable rents and transfers, Economics and Politics, 1, 17-40.

Hsu, Y.Y. and L.L. Wolf, 1999, The winner and loser effect: integrating multiple experiences, Animal Behaviour, 57, Part 4, 903-910.

Kaplan, T.R., I. Luski, and D. Wettstein, 2003, Innovative activity and sunk cost, International Journal of Industrial Organization, 21(8), 1111-1133.

Konrad, K.A., 2004, Inverse Campaigning, Economic Journal, 114 (492), 69-82.

Krishna, V., and J. Morgan, 1997, An analysis of the war of attrition and the all-pay auction, Journal of Economic Theory, 72 (2), 343-362.

Kura, T., 1999, Dilemma of the equality: an all-pay contest with individual differences in resource holding potential, Journal of Theoretical Biology, 
198 (3), 395-404.

Larsson, M., Beignon, A.S., and Bhardway, N., 2004, DC-virus interplay: a double edged sword, Seminars in Immunology, 16(3), 147-161.

Maskin, E. and J. Tirole, 2001, Markov perfect equilibrium I. Observable actions, Journal of Economic Theory, 100(2), 191-219.

Moldovanu, B. and A. Sela, 2001, The optimal allocation of prizes in contests, American Economic Review, 91 (3), 542-558.

Moldovanu, B. and A. Sela, 2004, Contest architecture, Journal of Economic Theory (forthcoming).

Müller, H.M., and K. Wärneryd, 2001, Inside versus outside ownership: a political theory of the firm, RAND Journal of Economics, 32(3), 527-541.

Organski, A.F.K., and E. Lust-Okar, 1997, The tug of war over the status of Jerusalem: Leaders, strategies and outcomes, International Interactions, 23 n(3-4), 333-350.

Parker, G.A., and D.I. Rubenstein, 1981, Role assessment, reserve strategy, and acquisition of information in asymmetric animal conflicts, Animal Behavior 29, 221-240.

Radner, R., 1992, Hierarchy: the economics of managing, Journal of Economic Literature, 30 (3), 1382-1415.

Radner, R.,1993, The organization of decentralized information processing, Econometrica, 61 (5), 1109-1146.

Runciman, S., 1987, A History of the Crusades, Cambridge University Press, Cambridge.

Sahuguet, N. and N. Persico, 2005, Campaign spending regulation in a model of redistributive politics, Economic Theory (forthcoming).

Schaub, H., 1995, Dominance fades with distance - an experiment on food competition in long-tailed macaques (macaca fascicularis), Journal of Comparative Psychology, 109(2), 196-202.

Tibbetts, E.A., and H.K. Reeve, 2000, Aggression and resource sharing among foundresses in social wasp Polistes dominulus, Behavioral Ecology and Sociobiology, 48(5), 344-352.

Wärneryd, K., 1998, Distributional conflict and jurisdictional organization, Journal of Public Economics, 69, 435-450.

Whitford, A.B., 2005, The pursuit of political control by multiple principals, Journal of Politics, 67(1), 29-49.

Yoo, C.Y., 2001, The Bush administration and the prospects of US-North Korean relations, Korean Journal of Defense Analysis, 13(1), 129-152. 
Zhou, D.P., C. Cantu, Y. Sagiv, N. Schrantz, A.B. Kulkarni, X.Y. Qi, D.J. Mahuran, C.R. Morales, G.A. Grabowski, K. Benlagha, P. Savage, A. Bendelac and L. Teyton, 2004, Editing of CD1d-bound lipid antigens by endosomal lipid transfer proteins, Science, 303 (5657), 523-527. 